\title{
Numerical Aperture of Single-Mode Photonic Crystal Fibers
}

\author{
Niels Asger Mortensen, Jacob Riis Folkenberg, Peter M. W. Skovgaard, and Jes Broeng
}

\begin{abstract}
We consider the problem of radiation into free space from the end-facet of a single-mode photonic crystal fiber (PCF). We calculate the numerical aperture $\mathrm{NA}=\sin \theta$ from the half-divergence angle $\theta \sim \tan ^{-1}(\lambda / \pi w)$ with $\pi w^{2}$ being the effective area of the mode in the PCF. For the fiber first presented by Knight et al. we find a numerical aperture , NA $\sim 0.07$ which compares to standard fiber technology. We also study the effect of different hole sizes and demonstrate that the PCF technology provides a large freedom for NAengineering. Comparing to experiments we find good agreement.
\end{abstract}

Keywords - Photonic crystal fiber, numerical aperture, Gaussian approximation

\section{INTRODUCTION}

$\mathrm{P}$ HOTONIC CRYSTAL FIBERS (PCF) constitute a completely new class of optical fibers consisting of pure silica with air-holes distributed in the cladding. Among many remarkable properties [1] PCFs are believed to have a potential for high-numerical aperture (NA) applications. Here we report a calculation of the NA for the class of PCFs first fabricated by Knight et al. [2], [3]. For this particular fiber we find a numerical aperture up to NA $\sim 0.07$. We also demonstrate how the NA may be controlled by the hole size for a given pitch and wavelength.

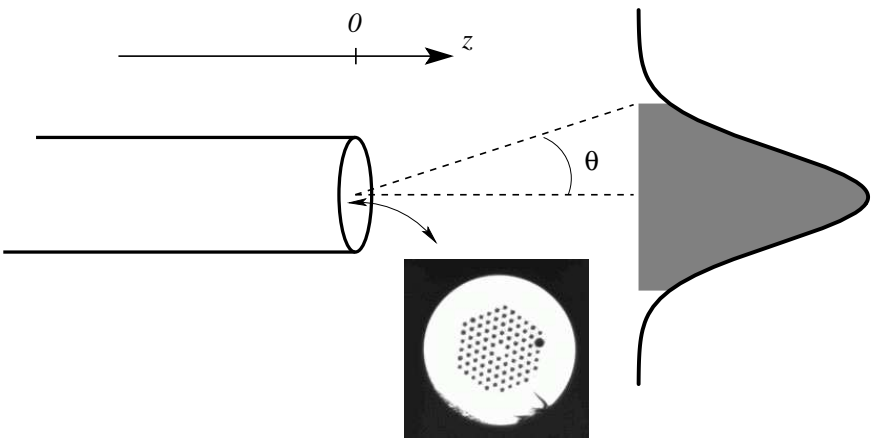

Fig. 1

COUPLing OF Light FROM END-FACET OF Fiber $(z=0)$ INTO FREE SPACE. The INSERT SHOWS A MICROGRAPH OF THE END-FACET OF A PCF.

The paper is organized as follows: First we consider the problem of radiation into free space from the end-facet of a single-mode optical fiber with a mode approximated by a Gaussian of width $w$. Solving the scattering problem at the end-facet of the fiber exactly we check the range of validity

The authors are with the company Crystal_Fibre A/S, Blokken 84, DK-3460 Birkerød, Denmark; http://www.crystal-fibre.com. of the text-book result $\theta \simeq \tan ^{-1}(\lambda / \pi w)$ (see $e . g$. Ref. [ $\mid$ | where $A_{\text {eff }}=\pi w^{2}$ is the effective area. For $w \ll \lambda$ we find deviations whereas nice agreement is found for $w>\lambda$. We then turn to the application of the PCF of Knight et al. [2] which belongs to the latter regime with $w>\lambda$. Finally, we compare our calculations to experiments.

\section{Numerical aperture in the Gaussian APPROXIMATION}

The numerical aperture NA $=\sin \theta$ (see Fig. 1) may be defined in various ways, but often one defines it in the far-field limit $(z \rightarrow \infty)$ from the half-divergence angle $\theta_{\nu}$ between the $z$-axis and the $\nu$-intensity point $r_{\nu}(z)$, i.e.

$$
\tan \theta_{\nu}=\lim _{z \rightarrow \infty} \frac{r_{\nu}(z)}{z}
$$

with $r_{\nu}(z)$ determined from

$$
\frac{\left|\Psi_{>}\left(z, r_{\perp}=r_{\nu}\right)\right|^{2}}{\left|\Psi_{>}\left(z, r_{\perp}=0\right)\right|^{2}}=\nu .
$$

For a Gaussian field $\Psi$ of width $w$ one has the standard approximate expression for $\nu=1 / e^{2} \simeq 13.5 \%$ [ ]

$$
\tan \theta_{1 / e^{2}} \simeq \frac{2}{k w}=\frac{\lambda}{\pi w} .
$$

For the $\nu=5 \%$ intensity point

$$
\tan \theta_{5 \%}=\sqrt{\frac{\ln 20}{2}} \times \tan \theta_{1 / e^{2}}
$$

which is often the one used experimentally. Eqs. (3, , 1 ) are valid for $k w \gg 1$, but in order to check the validity in the limit with $k w$ of order unity we solve the scattering problem at the end-facet of the fiber exactly. In the fiber $(z<0)$ the field is of the form

$$
\Psi_{<}(r) \propto \psi\left(r_{\perp}\right)\left(e^{i \beta(\omega) z}+\mathscr{R} e^{-i \beta(\omega) z}\right), z<0
$$

where the transverse field is approximated by a Gaussian

$$
\psi\left(r_{\perp}\right) \propto e^{-\left(r_{\perp} / w\right)^{2}},
$$

which has an effective area $A_{\text {eff }}=\pi w^{2}$ at frequency $\omega$. At the end-facet of the fiber $(z=0)$ the field couples to the free-space solution

$$
\Psi_{>}(r) \propto \int d \boldsymbol{k}_{\perp} \mathscr{T}\left(\boldsymbol{k}_{\perp}\right) e^{i \boldsymbol{k}_{\perp} \cdot \boldsymbol{r}_{\perp}} e^{i k_{\|} z}, z>0
$$


which is a linear combination of plane waves with $\omega=c k=$ $c(2 \pi / \lambda)$ and $\boldsymbol{k}=\boldsymbol{k}_{\perp}+\boldsymbol{k}_{\|}$.

In order to solve the elastic scattering problem, $\Delta \omega=$ $\omega(\beta)-\omega(k)=0$, we apply appropriate boundary conditions at the end-facet of the fiber; continuity of $\Psi$ and $\partial \Psi / \partial z$. At $z=0$ we thus get two equations determining the reflection amplitude $\mathscr{R}$ and the transmission amplitude $\mathscr{T}$. Eliminating $\mathscr{R}$ and substituting the resulting $\mathscr{T}$ into Eq. (7) we get

$$
\begin{aligned}
\Psi_{>}(r) \propto 2 \pi k^{2} & \int_{0}^{\infty} d \chi \chi \frac{2 n_{\mathrm{eff}}}{\sqrt{1-\chi^{2}}+n_{\mathrm{eff}}} \\
& \times e^{-(\chi k w / 2)^{2}} J_{0}\left(\chi k r_{\perp}\right) e^{i \sqrt{1-\chi^{2}} k z}
\end{aligned}
$$

Here, $\chi=k_{\perp} / k, J_{0}$ is the Bessel function of the first kind of order 0 , and $n_{\text {eff }}=\beta / k$ is the effective mode-index. Eq. (8) is the exact solution to the scattering problem and in contrast to many approximate text-book expressions (see e.g. Refs. (41) we have here treated the scattering problem correctly including the small, but finite, backscattering in the fiber. Thus, we take into account the possible filtering in transmitted $k_{\perp}$ at the fiber end-facet. The solution has similarities with the Hankel transform usually employed in the far-field inversion integral technique, see e.g. [5]. Numerically we have found that Eq. (8) gives a close-toGaussian field in the far-field limit.

In Fig. 2 we compare the two approximate solutions Eqs. (3,4) to a numerically exact calculation of $\tan \theta_{\nu}$ from Eq. (8). The calculation is performed for the realistic situation with $n_{\text {eff }}=\beta / k=1.444$ corresponding to a silicabased fiber. For $k w \sim 1$ the deviations increase because of the small, but finite, backscattering at the end-facet of the fiber. For $k w$ somewhat larger than unity a very nice agreement is found. A typical all-silica fiber like the Corning SMF28 has $k w>10$.

\section{APPLICATION TO PHOTONIC CRYSTAL FIBERS}

We consider the class first studied in Ref. [2] which consists of pure silica with a cladding with air-holes of diameter $d$ arranged in a triangular lattice with pitch $\Lambda$. For a review of the operation of this class of PCFs we refer to Ref. [6].

In applying Eq. (3) to PCFs we calculate $w$ from the effective area $A_{\text {eff }}=\pi w^{2}$ given by [7]

$$
A_{\mathrm{eff}}=\frac{\left[\int d \boldsymbol{r}|\boldsymbol{H}(\boldsymbol{r}, z)|^{2}\right]^{2}}{\int d \boldsymbol{r}|\boldsymbol{H}(\boldsymbol{r}, z)|^{4}} .
$$

Indeed we find that the corresponding Gaussian of width $w$ accounts well for the overall spatial dependence of the field. Of course we thereby neglect the satellite spots seen in the far-field [2], but because of their low intensity they only give a minor contribution to the NA [8].

For the field $\boldsymbol{H}$ of the PCF, fully-vectorial eigenmodes of Maxwell's equations with periodic boundary conditions are computed in a planewave basis [9].

Figure 3 illustrates the effective mode-index and effective area as a function of wavelength for a PCF with $d / \Lambda=$

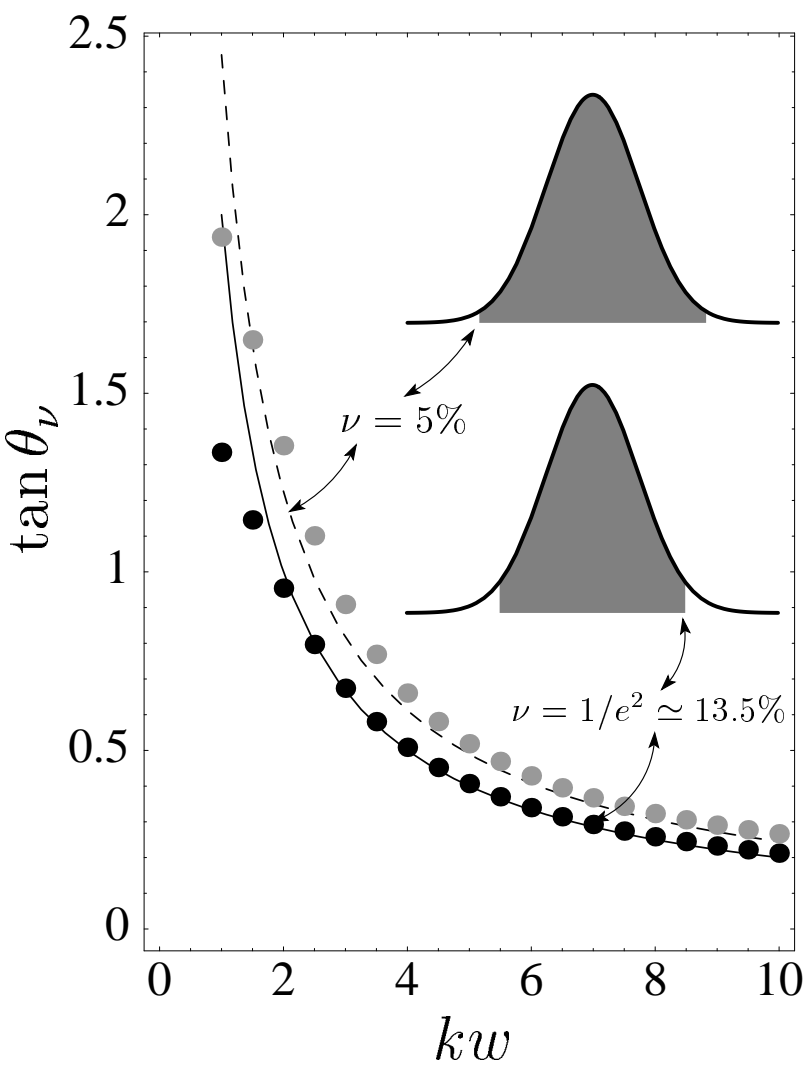

Fig. 2

Plot of $\tan \theta$ AS A FUNCTION OF THE DIMENSIONLESS PARAMETER $k w$. The points are the RESUlts of A nUMERICAL EXACT CALCUlation From EQ. (8) FOR A MODE With EFFECTIVE INDEX, $n_{\mathrm{eff}}=\beta / k=1.444$. The FUll AND DASHED Lines SHOW ThE APPROXIMATIONS EQS. (3, A), RESPECTIVELY.

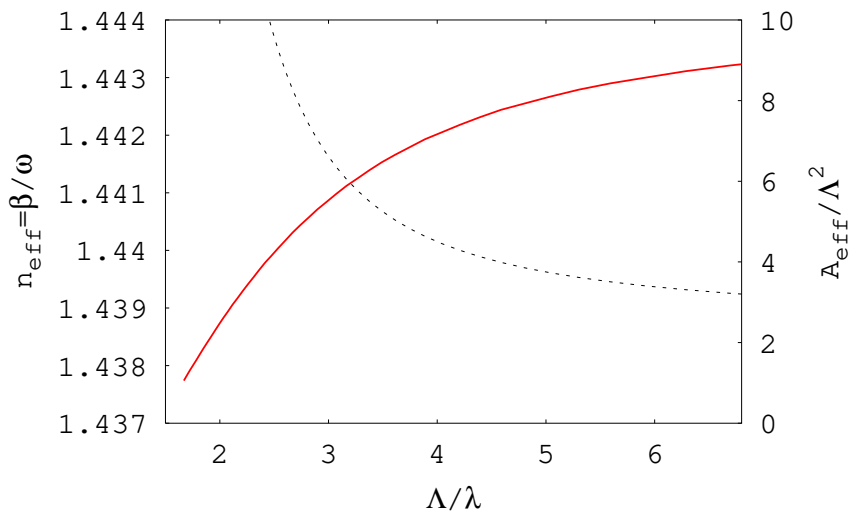

Fig. 3

EfFective mode-Index (SOlid Line, LEFT AXis) AND EFFECTIVE AREa (DAShed Line, Right AXIS) OF A PCF With $d / \Lambda=0.15$. 




Fig. 4

HALF-DiVERGENCE ANGLE (SOLID LINE, LEFT AXIS) AND $k w$ (DASHED LINE, RIGHT AXIS) OF A PCF with $d / \Lambda=0.15$.

0.15. The first PCF fabricated by Knight et al. [2] of this kind had a pitch of $\Lambda=2.3 \mu \mathrm{m}$ and was found to be single-mode in the range $\Lambda / \lambda$ between 1.5 and 6.8 . In Fig. 1 we show the corresponding half-divergence angle. We have also shown the value of the dimensionless parameter $k w$ (dashed line, right axis); the magnitude justifies the application of the approximate result in Eq. (3) to PCFs. We note that for non-linear PCFs 10] the value of $k w$ will approach the regime where deviations from Eq. (3) arise.

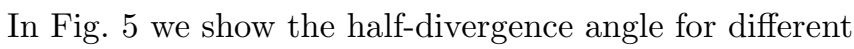
hole sizes where the fiber is endlessly single mode [11]. For small hole sizes $d / \Lambda$ we note that in practice the operation is limited by a significant confinement loss for long wavelengths where the effective area increases [12]. In Fig. 5 this can be seen as a bending-down of $\theta$ for small $\Lambda / \lambda$. In general the NA increases for increasing hole size and fixed pitch and wavelength. By adjusting the pitch $\Lambda$ and the hole size $d$ this demonstrates a high freedom in designing a fiber with a certain NA at a specified wavelength.

In order to verify our calculations experimentally a PCF with $d / \Lambda \sim 0.53$ and $\Lambda \simeq 7.2 \mu \mathrm{m}$ has been fabricated. In Fig. 6 we compare our calculations to a measurement of the NA at the wavelength $\lambda=632 \mathrm{~nm}$. As seen the calculation agrees well with the measured value.

\section{Conclusion}

We have studied the numerical aperture (NA) of photonic crystal fibers $(\mathrm{PCF})$. The calculations is based on the approximate "standard" result $\theta \simeq \tan ^{-1}(\lambda / \pi w)$ which we have found to be valid in the regime relevant to PCFs. As an example we have applied it to the fiber first fabricated by Knight et al. [2]. By studying the effect of different hole sizes we have demonstrated that the PCF technology have a strong potential for NA-engineering in the single-mode regime.

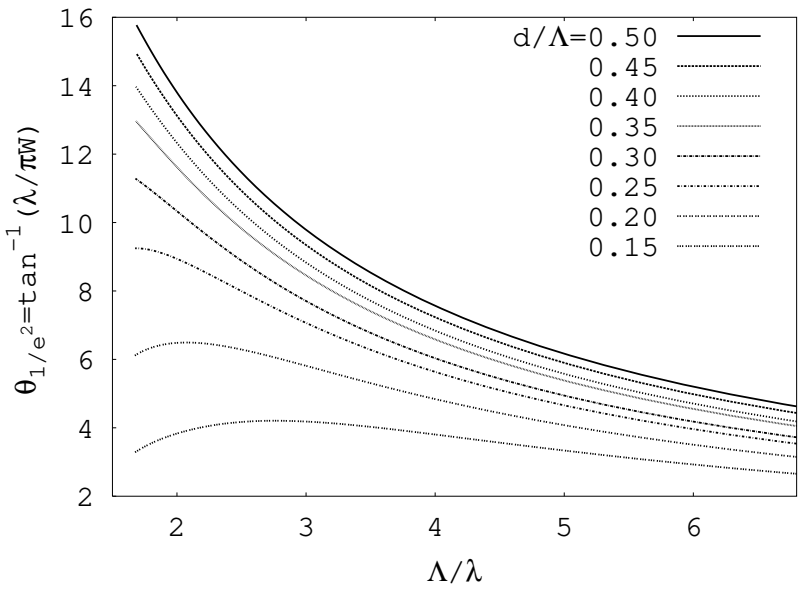

Fig. 5

HALF-Divergence ANGLE OF A PCF FOR DifFERENT HOLE Sizes.

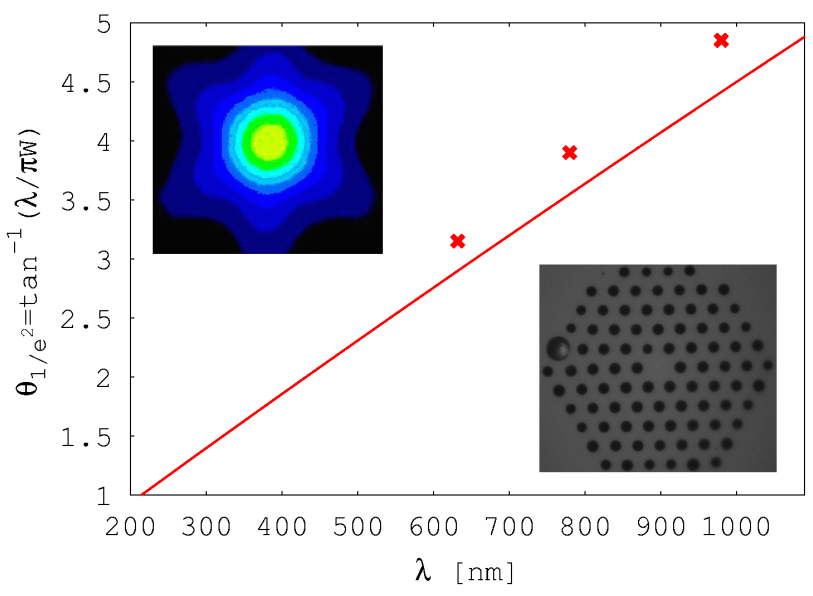

Fig. 6

HALF-DIVERGENCE ANGLE OF A PCF WITH $d / \Lambda \sim 0.53$ AND $\Lambda \simeq 7.2 \mu \mathrm{m}$. THE SOLID LINE IS A CALCULATION BASED ON THE IDEAL STRUCTURE AND THE DATA POINTS ARE MEASUREMENTS AT

$\lambda=632 \mathrm{~nm}, 780 \mathrm{~nm}$, AND $980 \mathrm{~nm}$ OF THE FIBER SHOWN IN THE LOWER Right InSERT. The UPPER LEFT INSERT SHOWS A NEAR-FIELD IMAGE AT $\lambda=632 \mathrm{~nm}$.

\section{REFERENCES}

[1] For recent special issues on photonic crystal fibers, see Opt. Express, vol. 9, pp. 674-779, 2001; J. Opt. A: Pure Appl. Opt., vol. 3, pp. S103-S207, 2001.

[2] J. C. Knight, T. A. Birks, P. St. J. Russell, and D. M. Atkin, "All-silica single-mode optical fiber with photonic crystal cladding," Opt. Lett., vol. 21, pp. 1547-1549, 1996.

[3] J. C. Knight, T. A. Birks, P. St. J. Russell, and D. M. Atkin, "All-silica single-mode optical fiber with photonic crystal cladding: errata," Opt. Lett., vol. 22, pp. 484-485, 1997.

[4] A. K. Ghatak and K. Thyagarajan, Introduction to Fiber Optics, Cambridge University Press, Cambridge, 1998.

[5] W. T. Anderson and D. L. Philen, "Spot size measurements for single-mode fibers - a comparison of four techniques," $J$. Lightwave Technol., vol. 1, pp. 20-26, 1983.

[6] J. Broeng, D. Mogilevstev, S. E. Barkou, and A. Bjarklev, "Pho- 
tonic crystal fibers: A new class of optical waveguides," Opt. Fiber Technol., vol. 5, pp. 305-330, 1999.

[7] G. P. Agrawal, Nonlinear Fiber Optics, Academic Press, San Diego, 2001.

[8] N. A. Mortensen and J. R. Folkenberg, "Near-field to far-field transition of photonic crystal fibers: symmetries and interference phenomina," Opt. Express, vol. 10, pp. 475-481, 2002.

[9] S. G. Johnson and J. D. Joannopoulos, "Block-iterative frequency-domain methods for Maxwell's equations in a planewave basis," Opt. Express, vol. 8, pp. 173-190, 2001.

[10] J. C. Knight, J. Arriaga, T. A. Birks, A. Ortigosa-Blanch, W. J. Wadsworth, and P. St. J. Russell, "Anomalous dispersion in photonic crystal fiber," IEEE Photonic Tech. L., vol. 12, pp. 807-809, 2000.

[11] T. A. Birks, J. C. Knight, and P. St. J. Russell, "Endlessly single mode photonic crystal fibre," Opt. Lett., vol. 22, pp. 961-963, 1997.

[12] T. P. White, R. C. McPhedran, C. M. de Sterke, L. C. Botton, and M. J. Steel, "Confinement losses in microstructured optical fibers," Opt. Lett., vol. 26, pp. 1660-1662, 2001. 